\title{
DOCUMENTATION
}

\section{Le Régulateur de pression Escher-Wyss pour turbines hydrauliques ${ }^{(1)}$}

Le régulateur de pression, ou vamne compensatrice, est un orifice qui s'ouvre en cas de décharge de la turbine, laisse instantanément échapper autant d'eau gu'il vient d'en être intercepté par la fermeture du distributeur, puis se referme automatiquement et lentement pour éviter toute surpression (coup de bélier).

La maison Escher-Wyss construit deux types de régulateurs de pression : à tiroir plat (fig. 1) ou tiroir rond (fig. 2). Le premier

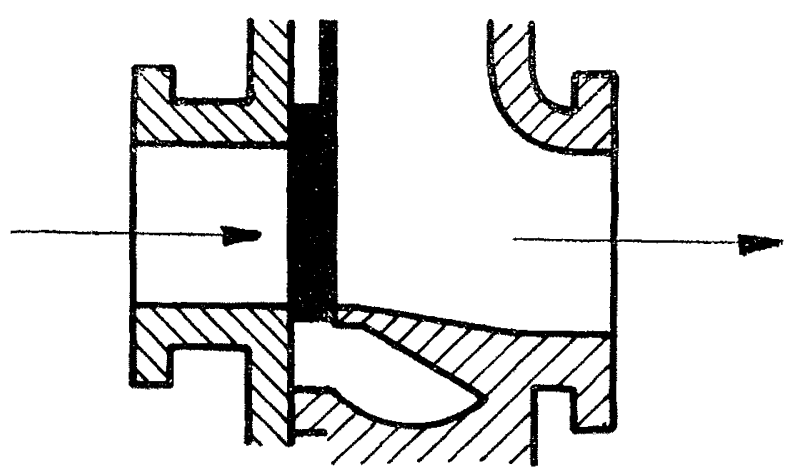

Fig. 1

s'emploie de préférence pour les hautes chutes et fables débits. Il présente l'avantage de refouler, pendant le fonctionnement les corps étrangers déposés sur la surface d'étanchéité, ce qui assure la parfaite étanchéité, si importante pour les chutes élevées.

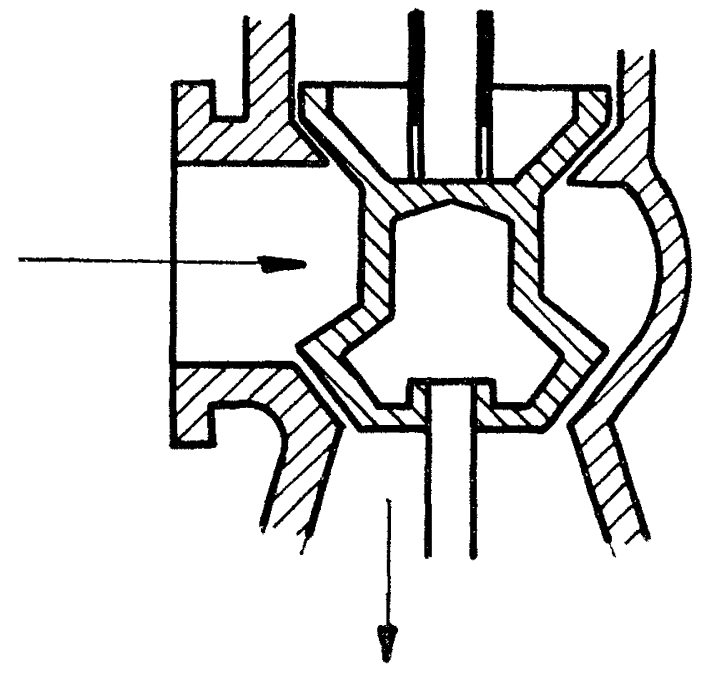

Fig. 2

En outre cet appareil évite le coude additionnel que comporte le second type.

I.e fonctionmement de l'appareil peut s'expliquer, comme il suit, à l'aide du schéma de la fig. 3. l'eau de la turbine accède au régulateur de pression par la tubulure A ct s'en écoule par la tubulure $\mathrm{B}$, normalement obturée par le tiroir C. Ce dernier se compose d'un corps surmonté d'un piston D, placé dans un cylindre, piston soumis par sa face supćrieure à la pression d'eau régnant dans la tubulure A. I'intérieur du cylindre E, communique par la conduite $F$ avec la pression constante d'eau dans

(1) D'après le Bulletin Escher-Wyss, Mai-Juin 1934, p. 75-77. l'espace A, par l'interposition d'un diaphragme G. L'espace E communique avec l'atmosphère par la tige creuse $T$. Lorsque l'appareil ne fonctionne pas, l'ouverture $H$ est fermée par l'obturateur I. Il règne alors dans l'espace $\mathrm{E}$ la même pression que dans l'espace $\mathrm{A}$ et le piston $\mathrm{D}$, ainsi que le corps du tiroir C, sont maintenus dans leur position inférieure.

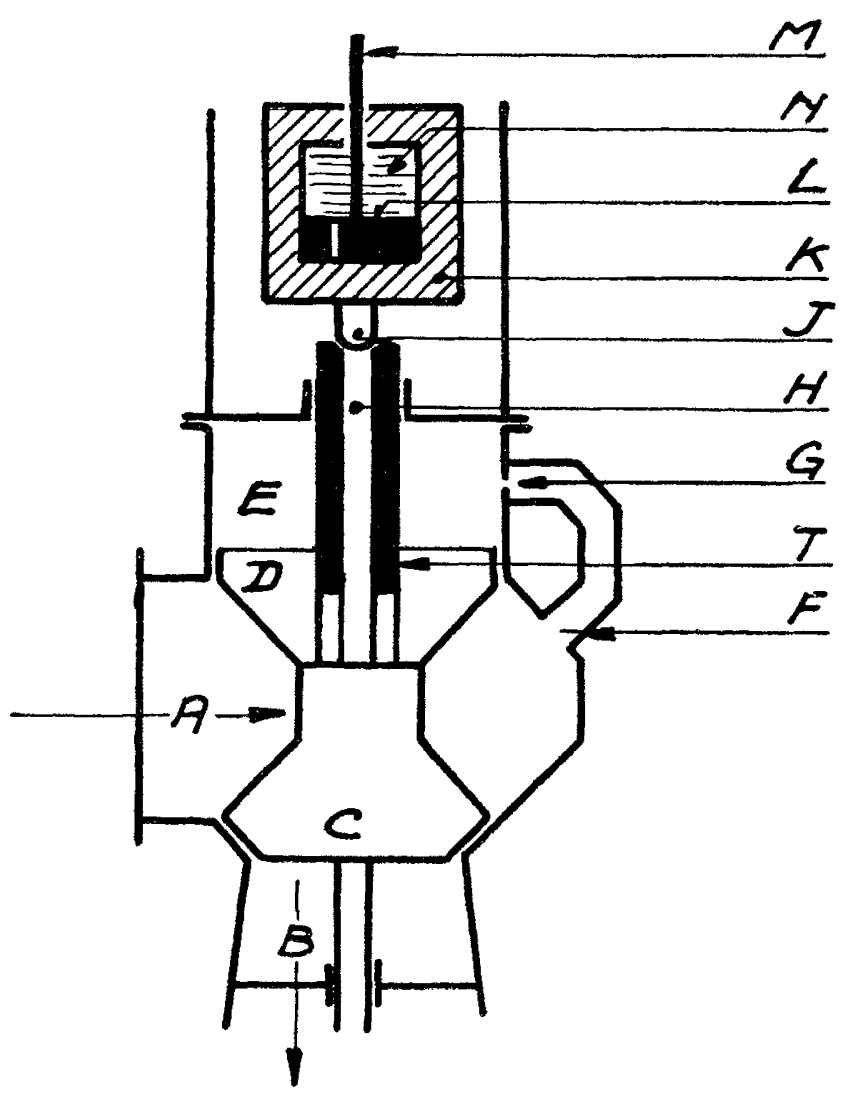

Fig. 3

L'obturateur $J$ est fixé au fond intérieur de la cataracte $K$. Cette dernière se compose d'un corps rempli d'huile, faisant office de poids dans lequel est logé le piston mobile $L$, qui comporte un diaphragme d'intercommunication de l'huile entre ses deux faces. La tige $M$ du piston est connectée au régulateur de vitesse, de façon qu'elle se soulève à chaque mouvement de fermeture, tandis qu'elle reste en repos à chaque mouvement d'ouverture.

Le soulèvement de la tige de piston $M$ entraìne le piston $L$ et par suite de la charge d'huile de l'espace $\mathrm{N}$, toute la cataracte $\mathrm{K}$ avec le tiroir. Liorifice $\mathrm{H}$ s'ouvre, la pression dans l'espace $\mathrm{E}$ diminue sensiblement par l'effet de l'étranglement provoqué par le diaphragme $G$, qui ne laisse passer que relativement peu d'eau. I.e piston D se soulève alors sous l'action de la pression constante d'cau agissant sur sa face inférieure, et le tiroir C s'ouvre clans la mesure correspondant au mouvement de lermeture de la turbine. Sous l'action du poids propre de la cataracte $K$, l'huile est refoulće de la chambre $\mathbf{N}$ sous le piston $\mathrm{L}$. La cataracte ainsi que le tiroir I redescendent lentement, en refermant l'ouverture $\mathrm{H}$ jusqu'à ce que la pression dans l'espace $E$ surpasse la contrepression venant du bas. Dès ce moment le piston $D$ et avec lui le tiroir $\mathrm{G}$ entraîné par le mouvement de la cataracte, s'abaissent jusqu’à la fermeture complète de l'orifice B.

L. B. 


\section{Le Régulateur Escher-Wyss à pression d'huile type $Z$ pour petites turbines hydrauliques ${ }^{(1)}$}

La masson Escher-II yss s'est proposée de réaliser un régulateur de type réduit, qui offre cependant toutes les possibilités que comporte un régulateur de grand type. Il doit en particulier permettre la marche entièrement automatique, done assurer la fermeture de la turbine, quelles que soient les conditions, mème si lactionnement du régulateur venait à faire défaut pour une cause quelconque.

La fig. 1 ci-contre représente le schéma simplifié de ce nouveau

\section{Fonetionnement.}

Supposons que la vitesse de la turbine s'élève par exemple. Lc pointean de réglage 12 s’abaisse, te cliaphragne 11 s'élargit, d'où une diminution de pression dans l'espace (9 el partant, daus ie crlindre 2. L'action du ressort devient prélominante, le pistons descend et actionne l'arbre 6 dans le sens de la lermeture. Ce mouvement provoque l'abaissemert du chemin de roulement 19 , done du levier 14 . La doulle de réglage 10 suit donc le mouvement

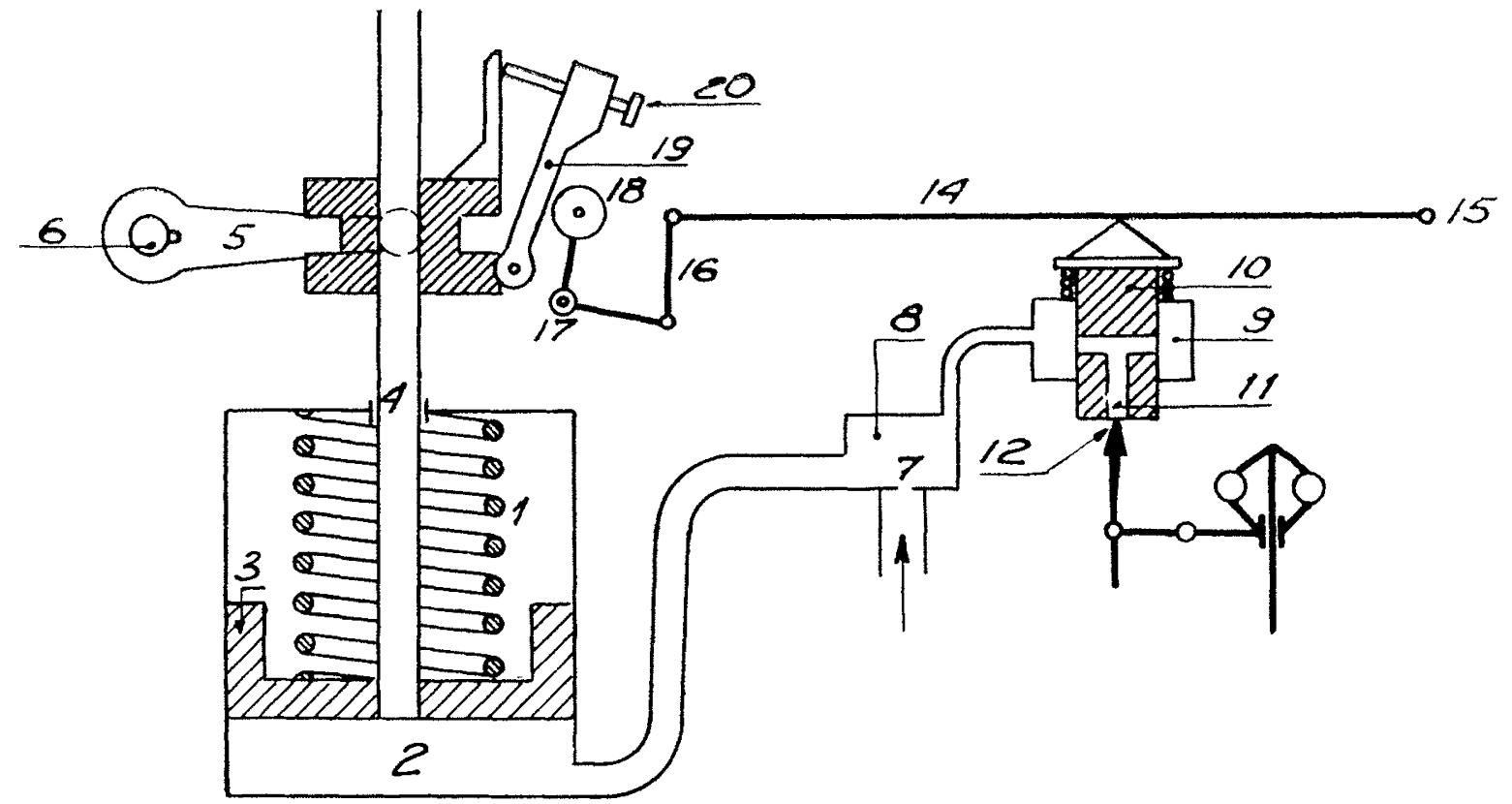

Fig I - Schéma simplifié du régulateur Escher-Wyss, lype $Z$ pour petites turbines hỵdrauliques

régulateur. L'énergie de fermeture est fournie par un ressort puissant 1, dont l'action est normalement écquilibrée par la pression d'huile qui règne dans le cylindre 2. Ce ressort agit sur l'arbre de réglage 6, par l'intermédiaire du piston 3 , de la tige 4 et du levier 5. L'huile sous pression est fournie par unc pompe à engrenages à travers un diaphragme 7 . La chambre de raccord 8 communiçuc, outre le cylindre 3 , avec la chambre sous pression 9. L'huile sous pression parvient ensuite à l'intérieur de la clouille de réglage 10 , pour en ressortir par le diaphragme 11 . Ce dernier est réglé par le pointeau 12, lui-même commandé par le tachymètre 13.

Le mécanisme d'asservissement comporte un levier principal 11 , qui peut osciller autour de l'articulation 15. L'extrémité de gauche de ce levier est reliée par la biellette 16 au levier oscillant 17, qui s'appuie au moyen du galet 18 sur le chemin de roulement 19. L'inclinaison de ce dernier peut ètre réglée par une vis 20.

\section{Le matériel électrotechnique}

Son

Le 21 février 1934, sous la présidence de M. P. Boucherot, M. Paul Brunet a fait une conférence sur ]'évolution du matériel électrique.

Après une allocution de M. P. Bovchepot, qui a rappelé la phrase assez mystérieuse qu'Eschyle met dans la bouche de Prométhée : "Les hommes trouveront un feu plus puissant que celui de la foudre ", et qui semble indiquer que les Grecs avaient des connaissances beaucoup plus complètes sur l'électricité qu'on ne le croit généralement, M. Paul Brunsar a montré l'évolution de l'électrotechnique depuis 1900.

C'est par la collaboration des techniciens de toutes les branches que l'on a vu progresser les réalisations anciennes el en apparaître de nouvelles. Les matières premières clle-mêmes ont évolué, propriétés magnétiques du fer, etc.

Les isolants ont largement évolué, les câbles ont suivi une marche parallèle, ainsi que les condensateurs. L'huile qui a eu toul d'abord un essort important subit un mouvement de recul du fait du danger d'incendie que présentent les masses importantes d'huile.

La meilleure qualité des matériaux employés dans les machines du pointeau de réglage 12 , jusçu'à ce clue la sortic du diaphragme 11 redevienne normale.

Jorsclue la vitesse baisse, le mouvement inverse se produit: le diaphragme 11 se ferme, la pression dans le cylindre '2 s'élève, le piston 3 monte en ouvrant l'admission.

La commande à la main est possible au moyen d'une roue à main montée sur le prolongement de la tige 4.

Tous les organes de ce régulateur, $y$ compris la pompe à engrenages, sont placés dans un bâti unique.

Cette disposition excessivement simple offre, assurent les constructeurs, toutes les possiblités que comporte un régulateur de grand type. Le degré d'irrégularité peut itre modifié en agissant sur l'inchanaison de la voie de roulement 19 , par la vis 20 . L.B.

(1) D'apres l'article de M. Gagg, dans le Bulletin Escher-Wyss de JuilletAoût 1931, p. 99-101.

\section{évolution et ses tendances actuelles}

tournantes a permis d'augmenter leur vitesse linéarre périphérique cui atteint $200 \mathrm{~m} / \mathrm{sec}$. Si l'on n'était pas limité par la fréquence actuelle de 50 périodes par seconde, on pourrait résoudre bien des problènes : meilleure utilisation des tôles et des condensateurs, chauflage par induction, pompes, compresseurs, etc.

Les rendements ont été considérablement améliorés par une diminution des pertes, dans le fer, daus le cuivre, par frottement, par ventilation.

Les lignes ont eu un immense développentent. L'inter-communication des réseaux a posé des prolslèmes nouveaux : stabilité, réglage, répartition de la charge.

La traction électrique se développe en France à la tension de 1500 V. en courant continu, ce qui est manifestement trop bas. Contrairement à l'iclée primitive, ce ne sont pas les lignes de montagne qui doivent être électrifiées les premières, mais celles à grand trafic.

L'appareillage a suivi lui aussi une évolution rapide, et iln'est pas juscu'au bruit des machines qui ne fasse l'objet des recherche actuclles.
La Technique Moderne. 15 avril 1934. 


\section{Le Régulateur automatique "Simplex" Cuénod pour machines à courant alternatif}

Le but poursuivi dans la conception de ce régulateur était une réalisation aussi économique que possible de l'appareil, tout en lui gardant une sensibilité et une rapidité satisfaisantes.

L'organe de mesure (vorr la figure) est constitué par un moteur

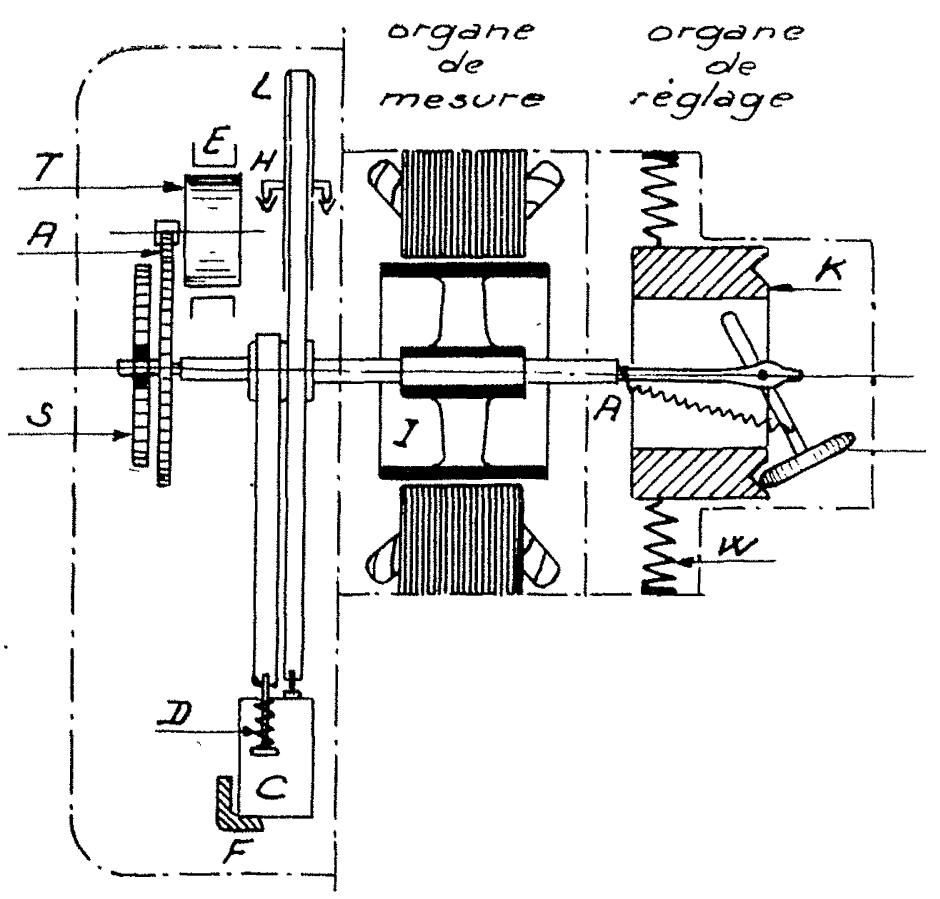

Fig. 1.- Schéma du régulateur "Simplex" Cuénod

Ferraris alimenté par le circuit à régler. Le couple exercé sur le rotor 1 est normalement équilibré par celui d'un contrepoids C. Ceci dans n'importe quelle position du rotor, de façon que le système est parfaitement astatique. L'axe A commande l'organe de réglage : une roulette $R$ en carbone spécial qui roule sans frotte- ment sur un collecteur $\mathrm{K}$, aux lames duquel sont reliées les résistances du réglage.

L'asservissement a pour but d'amortir le mouvement; l'axe A entraîne, par l'intermédiaire du ressort en spirale S et d'un train d'engrenages $B$, un tambour $T$, dont les mouvements sont freinés par les courants de Foucault qui s'y développent sous l'influence, d'ailleurs réglable, des aimants $F$.

Pour éviter les chocs, le rotor 1 est muni de butées élastiques à fin de course; d'autre part, le contrepoids C est suspendu par des ressorts $\mathrm{D}$, et repose, en cas de défaillance du couple moteur, sur des butées F solidaires du bâti.

Le fonctionnement est extrêmement simple : si, par exemple, la grandeur à contrôler devient inférieure à la valeur noımale, l'action du contrepoids devient prépondérante, l'axe $\mathrm{A}$, donc aussi, la roulette $R$, tournant dans le sens inverse des aiguilles d'une montre, et les résistances du rhéostat sont éliminées.

Limsensibilité du régulateur "Simplex " est de $\pm 0,5 \%$. La vitesse de réglage est sensiblement proportionnelle à l'écart à corriger, et pratiquement négligeable devant la constante de temps de la machine réglée elle-même. La consommation propre est de 80 voltampères. la tension maximum de service de 500 volts.

L'appareil peut être facilement muni de dispositifs complémentaires de statisme ou de compoundage, de facon que la grandeur contrôlée ne reste pas constante, mais varie suivant une loi convenable en fonction de la charge. Il convient au réglage d'une grandeur électrique alternative quelconque (tension, puissance, intensité, facteur de puissance, etc.), l'organe de réglage étant constitué par un rhéostat.

On cite à l'avantage de cet appareil, que, par un montage convenable, on a su éliminer les inconvénients inhérents au contrepoids : pesanteur et inertie, pour n'en garder que les avantages : astatisme absolu, simplicité et robustesse de construction. A cet effet, il n'est pas supporté par l'arbre de réglage $A$, mais repose sur les couteaux $H$ de la balance $L$. Ses accélérations sont calculées suffisamment faibles pour que l'inertie du système devienne néglıgeable.

I. B.

\section{Les coups de bélier et leurs conséquences - Méthodes et dispositifs permettant de les éviter}

Au moment de l'arrêt d'une pompe, il se produit une surpression qui se propage dans la conduite et qui, ayant une forme dynamique, rique de provoquer des efforts correspondant à une pression statique double, soit 3 fois la pression de marche.

Plusieurs moyens permettent d'éviter ces surpressions :

$1^{0}$ L'Emploi de vannes à fermeture lente. Dans ce cas la pompe sc met à for.ctionner en turbine et il faut prévoir des appareils automatiques délicats ;

$2^{\circ}$ On place sur les conduites des soupapes de sûreté. Parson principe ce système ne fonctionne que s'il $y^{\prime}$ a surpression : il ne la supprime pas. Étant domé la vitesse de propagation de l'onde $(1.400 \mathrm{~m} / \mathrm{s})$ el l'inertic des soupapes cette protection est illusoire;

$3^{\circ}$ Cheminées al'équilibre. Elles ne protègent pas le tronçon entre la pompe et la chemince d'équilibre. Au delà la réduction est proportionnelle au rapport entre la section de la conduite et la section totale : cheminée + conduite;

$4^{\circ}$ On place un réservoir d'air sur la conduite. Mais il doit avoir de très grandes dimensions et s'il est mal calculé, il peut augmenter le coup de bélier; $5^{\circ}$ On détruit par laminage la totalité de l'énergie cinétique de la colonne d'eau, avant l'apparition possible du coup de bélier.

Le coup de bélier ne peut en effet apparaître qu'au bout d'un temps supérieur au double de la longueur de la conduite, divisé par la vitesse de propagation $(1.400 \mathrm{~m}$.).

La conduite est munie d'un réservoir d'air relativement petit, constituant surtout une réserve d'eau. Ce réservoir communique avec la conduite par un orifice formant tuyère calibrée.

En cas d'arrèt de la pompe, le clapet de retenue se ferme immédiatement, tandis que la colonne d'eau continue son mouvement du fait de son inertie. C'est alors le réservoir qui fournit à la conduite le volume d'eau correspondant à son débit. Le laminage dans la tuyère diminue considérablement la pression dans la conduite. Bien entendu la section de la tuyère doit ètre rigoureusement calculée.

Une installation ainsi réalisée à la station de pompage d'Albi a supprimé complètement les coups de bélier au moment de l'arrèt des pompes.

\section{Avignon.}

La Technique Moderne. $1^{\mathrm{er}}$ avril 1934. 


\section{L'Usine hydro-électrique d'Orsières, en Valais \\ par la Société Suisse d'Electricité et de Traction de Bâle}

L'usine d'Orsieres, située au confluent des Drances de Ferret et a'Entremont, utilise l'eau de ces deux torrents sous une chute brute d'environ 400 mètres.

La concession en avait été donnée en 1900 à deux particuliers qui la cédèrent en 1906 à la Compagnie des Forces Motrices d'Orsières, qui commenca les travaux en 1908. Ceux-ci ne furent exécutés que par intermittence jusqu'en 1929 date à laquelle les actions de cette société furent rachetées par la Sociétí pour l'Industrie chimique et la Société Suisse d'Électricité et de Traction. Cette dernière fut chargée des travaux qui furent poussés activement et le 15 aout 1931 l'usine entra en fonctionnement.

Le projet primitif ne fut pas très modifié pour tenir compte des travaux déjà réalisés et par suite de la nécessité de reprendre immédiatement les travaux, sous peine de perdre la concession.

Le bassin versant de la Drance d'Entremont à Pallazuit, où se trouve le barrage, est de $110 \mathrm{Km}^{2}$ : celui de la Drance de Ferret à Branche, où est l'autre barrage, de $70 \mathrm{Km}^{2}$. Par leurs effuents, qui viennent des glaciers leur débit a une allure torrentielle et alpine, faibles clébits en hiver et forts débits en été.

Le débit actuel de $t \mathrm{~m}^{3} / \mathrm{sec}$, de pleine charge de l'usine, correspondant à $22 \mathrm{l} / \mathrm{sec}$. par $\mathrm{Km}^{2}$ de bassin versant, est assuré pendant 6 mois de l'année, du $1^{\mathrm{er}}$ mai au $1^{\mathrm{er}}$ septembre; il correspond à une puissance utile aux bornes des alternateurs de $12200 \mathrm{~kW}$. Le débit utile d'étiage tombe normalement à $1200 \mathrm{l} / \mathrm{sec}$, capables de fournir une puissance utile de $4000 \mathrm{~kW}$. L'énergie disponible annuellement s'élève à $86.000 .000 \mathrm{kWH}$, dont 36.000 .000 d'énergie constante.

Les ouvrages de prise d'eau de Pallazuit sur la Drance d'Entremont et ceux de Branche sur la Drance de Ferret sont semblables. Ces barrages d'une trentaine de mètres de longueur sont constitués par un simple mur de $1^{\mathrm{m}} 50$ de hauteur, auquel fait suite, à l'aval, un radier fortement incliné en moellons de granit.

On s'aperçut que les radiers à l'aval des barrages avaient été fortement affouillés. On en assura le pied par de gros blocs de granit, enrobés dans une couche épaisse de béton, réalisant ainsi une imitation du lit naturel du torrent. Cette disposition a donné de très bons résultats.

Pour assurer la décantation des eaux on a installé à chaque prise d'eau, un dessableur système Dufour logé dans le tunnel de la galerie.

Les galeries sont creusées en majeure partie dans des schistes calcaires, gréseux on marneux, qui constituent les deux versants du Val Ferret et une bonne partie du versant gauche du Val d'Entremont.

Les galeries, exécutées à une époque où les moyens mécaniques n'étaient pas d'un usage courant, ont été creusées à la main, sans aucune ventilation artificielle, c'est ce qui explique les nombreuses fenêtres, distantes tous les $300 \mathrm{~m}$. et de 30 à $50 \mathrm{~m}$. de profondeur. Les galeries sont donc situées dans des couches peu profondes, soit dans la moraine, soit dans des roches voisines de celles qui sont soumises au fauchage, ou balancement superficiel des couches.

Il fallut donc consolider ces galeries par des nettoyages, des revêtements et des injections de ciment (1).

Pour éviter des bancs de gypse sur la rive droite de la Drance de Ferret, le flanc gauche de la vallée a été emprunté sur une longueur de $1500 \mathrm{~m}$. à partir de la prise d'eau, jusqu'à la Reuse de Saleinaz, où le flanc droit est rejoint par un siphon. Ce siphon facilitera en même temps l'adduction de la Reuse de Saleinaz envisagée pour plus tard.

Le siphon de Saleinaz est constitué par un tube métallique de $1^{\mathrm{m}} 40$ de diamètre et de $1.380 \mathrm{~m}$. de longueur.

(1) La description de ces travaux forts intéressants ne peut être résumée sans perdre toute valeur. Voir à ce sujet le No 7, 31 Mars 1934, du Bulletin Technique de la Suisse Romande.
Pour éviter Ia formation de glace sur ses parois en hiver, où le débit tombe à $0,5 \mathrm{~m}^{3 /} / \mathrm{sec}$. et la vitesse à $0,35 \mathrm{~m}^{3} / \mathrm{sec}$, le lube est enterré sous $1 \mathrm{~m}$. de remhlais. Il franchit la Drance dans une passerelle en béton armé, formant caisson. La tôle a une épaisseur de 7 à $10 \mathrm{~m} / \mathrm{m}$. Pour éviter les déformations sous le poids des déblais, les tuyaux sont renforcés tous les $2 \mathrm{~m}$. par des frettes en cornieres de $100 \times 65 \times 11$.

Les deux branches du siphon prennent appui sur deux massifs d'ancrage. situés de part et d'autre de la passerelle. La partie horizontale du siphon, dont les extrémités sont maintenues par les massif's d'ancrage est munie d'un dispositif d'expansion. Au point le plus bas est installée une vamne de purge.

Des deux chambres de mise en charge primitives, celle dite de Lidkes, moins exposée aux déplacements a seule été conservée. De nouvelles fissures ayant ćté constatées en 19:30, on fut amené à remplir les vides laissés par less premiers travaux, à consolider le rocher ébranlé par le fauchage superficiel et les nouveaux travaux, et par remplissage et injection de ciment, à renforcer la chambre elle-mème, en armant ses parois et en établissant des drains pour l'évacuation des infiltrations éventuelles.

La chambre mème se compose de deux compartiments. Les excédents d'eau se déversent dans un couloir central, aucquel fait suite la conduite de trop plein. Des vannes de fond permettent de meltre à sec l'un ou l'autre des compartiments sans interrompre le service.

Le fait davoir intercalé un tronçon de galerie en pression, entre la partie à écoulement libre et la mise en charge, semble avour supprimé tout phénomène d'oscillation des ondes liquides, que l'on observe fréquemment dans les galeries à écoulement libre.

Le trop plein est réalisé par une concluite d'acicr de $80 \mathrm{~cm}$. de diamètre et de $7 \mathrm{~mm}$. (l'épaisseur, parallèle à la conduite forcée. Elle est munic de 18 reniflards de $30 \mathrm{~cm}$. rle diamètre, et aboutit à un diffuseur placé en face de l'usine sur la rive droite de la Drance. La forme et la dimension du diffuseur furent déterminées à la suite d'essais sur modèle. L'embouchure verticale est troncconique, percée latéralement de trous circulaires. La forme et les chicanes de l'embouchure jouent un rôle prépondérant sur l'amortissement de l'eau.

Les reniflards, disposés le long de la conduite, ont pour but de permettre l'aspiration ou le rejet de l'aur par l'cau en écoulement libre. A la partie inférieure de la conduite les reniflards sont munis de soupapes à contrepoids réglables qui se ferment automatiquement dès que l'cau atteint la soupape et s'ouvrent lorsque le niveau de l'eau baisse.

Près de l'usine la concluite forcée. qui avait suivi un tracé rectiligne, décrit un coude de $130^{\circ}$ et se lermine par le collecteur placé dans le sous-sol de l'usine. Tout est prévu pour que l'on puisse placer la deuxième conduite et la raccorder sans aucune interruption de service.

La concluite a une longueur de $860 \mathrm{~m}$. et un diamètre variant de $1^{\mathrm{m}} 10$ à $1 \mathrm{~m}$. L'épaisseur des tôles varie de 7 a $25 \mathrm{~mm}$. Sur place les tuyaux ont été raccordés entre cux par soudure électrique, suivant le procédé Sulzer à 2 lignes de soudure. Pour faciliter la mise en place on a placé des joints de dilatation au-dessous des ancrages, condamnés après coup par soudure.

La vanne d'admission d'eau est cylindrique à commande automatique, depuis la centrale, doni la fermeture est réglée par un frein centrifuge.

Un radier de gros blocs de granit, enrobés dans une forte couche de béton, présentant la même rugosité que le lit naturel de la rivière, a été étab)]i au-dessus de la galerie qui renferme la conduite, au passage sous la Drance. Jusqu'à présent on n'a pas constaté d'affouillement au droil de la galerie, phénomène qui n'aurait pas manqué de se produire avec un radier lisse.

Le canal de fuite de $125 \mathrm{~m}$. de longueur est muni d'une chambre de jaugeage. 
Jien que le sol de l'usine se trouve au-dessus du niveau des plus hautes crues connues, pour parer à tout danger d'inondation la largeur du lit de la Drance, au droit de l'usine, a été presque doublée.

I'usine est équipée de deux groupes utilisant chacun $2 \mathrm{~m}^{3} / \mathrm{sec}$. I'emplacement d'un tioisième groupe est déjà réservé et on pourra éventuellement en installer un quatrième, Je débit total utile atleignant alors $8 \mathrm{~m}^{3} / \mathrm{sec}$, correspondant à celui des deux conduites forcées en parallèle, et à celui de la conduite de trop plein.

Les groupes sont à axe vertical, afin de pouvoir placer les turbine's sullsamment bas pour restituer l'eau directement dans la retenue de l'usine de Sembrancher.

L.es turbines Pelton de 9.100 CV. à 600 tours/minute, ont une rone de $1.250 \mathrm{~mm}$. de diametre et deux injecteurs. Sous $383 \mathrm{~m}$. de chute nette, le débit à pleine ouverture est de $2.170 \mathrm{l} / \mathrm{sec}$. Sur chaque tubulure des injecteurs sont une vanne à tiroir et une vanne sphérique, la première ne servant qu'en cas de réparation.

Le régulateur et tous les organes de commande sont dans la salle des machines. Le servo-moteur des pointeaux est, par contre, placé près de la turbine elle-mème. Dans un but de sécurité, le régleur, du typo accéléro-tachymétrique des Atelıers des Charmilles, est commandé par un alternateur-pilote de $1.5 \mathrm{kVA}$, placé en bout d'arbre de l'allernateur, au-dessus de l'excitatrice.

Cet alternateur-pilote a deux enroulements indépendants l'un pour l'excitation, l'autre pour la production de courant alternatıf triphasé à 20 périodes. La tension de l'alternateur-pilote varic proportiomellement à la vitesse pour atteindre $160 \mathrm{~V}$. à $60)(0$ tours.

Le moteur du régleur ne se met en marche que pour une vitesse de 32.5 tours/minute ef il ne se décroche que vers 180 tours/minute.

Les alternateurs triphasés ont une tension de $10500 \mathrm{~V}$. et une fréquence de 50 périodes/sec. I.eur puissance apparente est de $7000 \mathrm{kVA}$. Les excitatrices shunt ont une puissance de $56 \mathrm{~kW}$. Le rotor forme lui-mème ventilateur. L'ne ouverture permet d'aspirer l'air dans la salle des machines, pour créer en hiver une circulation de chauffage. Sur les cheminées de refoulement d'air chand se trouvent des jalousies, permettant d'envoyer l'air chaud pour le chauffage des divers locaux.

Presque toute l'énergie étant transportée à grande distance, on a réalisé la liaison directe alternateur-transformateur. de facon à former en qquelque sorte des unités de $50 \mathrm{kV}$. Pour les services locaux et l'alimentation du réseau $10 \mathrm{kV}$. d'Orsières, on a placé sur le câble de liaison de l'alternateur au transformateur, une

\section{A propos des}

Ia catastrophe du Lac Noir (1) a suggéré à M. L. DU Bors, sur les vannes-papillons, des réflexions insérées dans les $n^{08} 5$ et 6 , 3 et 17 mars 1934 du Bulletin Technique de la Suisse Romande, dont voici un résumé succinct

Les vannes-papillons sont des organes de fermeture très simples, utilisés en hydraulique depuis longtemps. Dans la position fermée les poussées hydrauliques sur la face amont du papillon s'équilibrent exactement et l'on n'a à vaincre, au début de l'ouverture, que les frottements dans les tourillons.

Dans la position ouverte en plein, l'équilibrage est également réalisé. Par contre il n'en est pas de même dans les positions intermédiaires. Pour celles-ci les poussées sur la face amont du papillon vont en diminuant dans le sens du courant de l'eau, à cause de la vitesse croissante de la masse liquide.

La poussće résultante est désaxée et agit sur le papillon avec un bras de levier produisant un moment de torsion. Ce noment de torsion lend à fermer le papillon. En cas de rupture en aval, lorsque le débit augmente beaucoup les efforts de fermeture peuvent devenir considérables. Le calcul exact des poussées n'est pas aisé; on peut toutelois, par des calculs approximatifs, se rendre compte de leur ordre de grandeur.

Dans le cas où l'écoulement se fail à gueule bée, c'est-à-dire, lorsqu'il se produit en aval du papillon une rupture importante de la conduite et que l'on peut compter que la pression sur la face aval du papillon est voisine de la pression atmosphérique, le moment de torsion, exprimé en tonnes-centimètres, a pour valeur :

$$
\mathrm{M}_{\mathrm{t}}=\mathrm{K} \cdot h \cdot \mathrm{F} \cdot r
$$

(1) Voir La Houille Blanche, No 205-206, Janvier-Février 1934. cellule rlite "cellule d'alternateur. "C'est dans ces cellules que l'on a formé le point neutre des alternateurs avec résistance de mise à la terre.

Pour la partie à $50 \mathrm{kV}$. l'appareillage est à l'intérieur et les transformateurs à l'extérieur. Les disjoncteurs $50 \mathrm{kV}$. sont encastrés dans le plancher, l'étage inférieur étant aménagé en chambre d'explosion, avec ouvertures directes vers l'extérieur. Les transformateurs de $7.000 \mathrm{kVA}$. ont leur point neutre mis à la terre par l'intermédiaire d'une bobine d'extinction.

La salle des tableaux, située au premier étage, a vue sur la salle des machines, par une large baie vitrée. Les services auxiliaires sont alimentés par deux transformateurs de $150 \mathrm{kVA}$.

I'énergie est surtout destinée aux usines de Monthey de la Société pour l'Industrie chimique à Bâle. Il n'a été projeté cu'une seule ligne à deux circuits dont un seul a été équipé pour le moment. I'écartement des conducteurs a été prévu pour que l'on puisse éventuellement porter la tension à $120 \mathrm{kV}$. La ligne a été tirée jusqu'à Vernayaz, où elle se raccorde avec une ligne existante à $50 \mathrm{kV}$. venant du Haut-Valais et se dirigeant également vers Monthey.

Le tronçon " montagne " comprend deux secteurs l'un allant d'Orsières à Sembrancher, équipé de portiques à 9 conducteurs, l'autre de Sembrancher à Martigny, équipé de pylônes du type courant pour 6 conducteurs. Pour augmenter la sécurité des supports, en annulant presque complètement tout effort de torsion, en cas d'efforts unilatéraux, les consoles sont prévues tournantes autour d'un axe, qui est aussi rapproché que possible de la face du fut.

La porté moyenne est de $300 \mathrm{~m}$. ; la hauteur moyenne du câble de terre au-dessus du sol d'environ $25 \mathrm{~m}$. Les conducteurs aluminium-acier ont une section totale de $188 \mathrm{~mm}^{2}$. Pour la descente dans la vallée du Rhône, la proportion d'acier a été élevée à $40 \%$.

Les isolateurs sont du type cape et tige. Il est prévu 4 éléments de $280 \mathrm{~mm}$. de diamètre et $170 \mathrm{~mm}$. de hauteur pour le passage du Mont Chemin et 3 éléments pour le reste de la ligne.

Le raccordement de Vernayaz n'est que provisoire. Les postes d'Orsières et de Monthey sont équipés de postes téléphoniques à ondes dirigées. A Monthey se trouve un auto transformateur à gradins à commande automatique pour régler la tension. Il est d'une puissance traversante de $10.000 \mathrm{kVA}$.

\section{Bulletin Technique de la Suisse Romande.}

3 mars, 31 mars, 28 avril, 12 mai, 9 juin, 7 juillet, 21 juillet 1934 .

\section{vannes-papillons}

dans laquelle :

$h=$ pression en m., en amont;

$\mathrm{F}=$ surface $\mathrm{du}$ papillon, en $\mathrm{m}^{2}$

$r=$ rayon du papillon, en $\mathrm{cm}$;

$\mathrm{K}=$ un paramètre dont la valeur maximum, pour la position la plus défavorable serait de 0,145.

Si le papillon est intercalé sur une conduite, il faut mettre au lieu de $h$, la valeur $h-h_{1}, h_{1}$ étant la pression qui s'établit entre le papillon et l'orifice d'écoulement à l'extrémité de la conduite.

$$
\mathbf{M}_{\mathrm{t}}=\mathrm{K}\left(h-h_{\mathrm{1}}\right) \mathrm{F} \cdot r
$$

$h_{1}$ dépend de la position du papillon. Soit $f_{1}$ la section d'écoulement variable du papillon, $f_{2}$ la section théorique de l'orifice d'écoulement au bout de la conduite, lorsque le papillon sera ouvert en plein. En négligeant le coefficient de contraction.

$$
h_{1}=h \cdot \frac{f_{1}^{2}}{f_{1}^{2}+f_{2}^{3}}
$$

En faisant varier $f_{1}$ de 0 à sa valeur maximum, on peut ainsi déterminer le moment de torsion pour les diverses positions.

Il serait très intéressant de vérifier expérimentalement la valeur du moment de torsion, pour diverses positions du papillon et pour différents débits, dans une installation existante. On pourrait ainsi déterminer les valeurs expérimentales du paramètre $K$.

L. DU BoIs. 


\section{La sous-station de Bleiloch des Thüringenwerke A.-G., Weimar}

I a société "Obere Saale, Weimar " a mis en service fin 1932 la centrale de Bleiloch alimentée par le premier des deux grands barrages projetés sur le cours supérieur de la Saale.

Sa puissance de $10.000 \mathrm{~kW} / 50.000 \mathrm{kVA}$ est répartic sur deux unités comprenant chacune deux turbines, une pompe et deux alternateurs moteurs.

La sous-station de Blciloch sert non senlement ì transmettre l’énergie produite par la centrale, mais encore à lui fournir l'énergie qui lui est nécessaire. Iorcpu'elle fonctionne en pompage pour laccumulation.

La tension de $100 \mathrm{kV}$. est nécessaire pour l'intercommexion avec le réseau de la Sàxe, par le poste de Herlasgrun, celle de $50 \mathrm{hV}$. pour le réseau de Thuringe el celle de $10 \mathrm{kV}$. pour le couplage avec la petite usine de Burghhammer, travaillant au fil de l'eau, à la sortie du réservoir d'égalisation et située à $1 \mathrm{~km}$. en aval de Bleiloch $(1.000 \mathrm{~kW} / 1250 \mathrm{kVA}$ en première itape, le double en construction définitive). Les services auxuliaires et la distribution locale sont également sur le côté $10 \mathrm{kV}$.

Chacun des deux groupes générateurs $10 \mathrm{kV}$. alimente un transformateur. Ces transformateurs à trois enroulements $10 / 50 / 100 \mathrm{kV}$. servent au transport et à l'échange de l'énergic entre les côtés 50 et $100 \mathrm{kV}$. Pour cette rason il a fallu prévoir un disjoncteur entre l'alternateur et le transformateur.

Pour le côté $100 \mathrm{kV}$., il sullt d'un smple jeu de harres. car il n'y a que deux arrivées de transformateurs et deux départs de ligne. Un sectionnement avec disjoncteur permet la marche indépendante des deux groupes.

Sur le $50 \mathrm{kV}$. les besoins de couplage sont plus variés et l'on a prévu un double jeu de barre, avec la possibilité d'installer un transformateur de réglage afin de faire varier la répartition de l'énergie active clans la boucle forméc par l'interconnexion des côtés 50 et $100 \mathrm{kV}$.

La distribution à $10 \mathrm{kV}$. est indépendante du côté des machines, à cause des grosses variations de tenisions 9 et $11 \mathrm{kV}$. entre la marche en moteur et la marche en générateur, et du fait de la nécessité de rester continuellement en service.

Pour la sous-station il fallait tenir comple de conditions particulières :

emplacement de $2.000 \mathrm{~m}^{2}$, d'une surface très irrégulière, escarpée de tous les côtés;

humidité considérable, hiver long avec beaucoup de neige, orages très fréquents;

couplages quotidiens fréquents, intervention certaine ct à n'importe quel moment, comme usine de secours.

On se décida alors en faveur d'un poste intérieur muni de disjoncteur à expansion, qui entraînaient une économie de place.
Les postes 50 et $100 \mathrm{kV}$. sont logés chacum dans deux halls séparés, disponés de part et d'autre du bàliment central de commancle, et sur le mème niveau. Les deux halls sont des pièces simples sans cellules, ni parois intermédiaires.

Toutes les commandes sont pneumatiques, pour les sectionneurs, comme pour les disjoncteurs. et mème pour certaines fenètres difficilement accessibles.

Etant domme la grande humidité et la frécpuence des orages tous les isolateurs sont cu matière c'éramique.

Le batiment central comprend la salle de commande, et les locaux pour hatterie $220 \mathrm{~V}$. avec redresseur à oxyde de cuive, le poste dair comprimé, la téléphonie à haule fréquence, les relais, les eompteurs. le poste de $10 \mathrm{kV}$. et les services auxiliaires B'T.

L'installation $10 \mathrm{kV}$. ne comprend aucun matériel contenant de l'hule. Les transformateurs fonctionnent à sec, et les disjoncteurs sonl du trpe à expansion. Ja salle des commantes est séparée de linstallation $10 \mathrm{kV}$. par une paroi en verre et le tableau des compleurs.

Le tableau comporte un schéma synoptique avec indicateur de positions électromannétıques. Par contre tout défaut est signalé par une lampe, comme par exemple l'ouverture automatique d'un disjoncteur.

La marche des deux groupes de la centrale est commandée du poste. J.es mancuvres hydrauliques et mécaniques sont surveillées du poste au moyen de transmetteurs d'ordres.

Les câbles $10 \mathrm{kV}$. provenant de la centrale aboutissent dans des armoires, placées à l'extérieur et renfermant des disjoncteurs à expansion. Les transformateurs sont immédiatement à côté sur le terre-plein.

Chacun des trois enroulements est prévu pour la pleine puissance du groupe, $25 \mathrm{MVA}$. Ils sont munis de prises $\pm 6 \%$ sur le côté $100 \mathrm{kV}$. et d'un coupleur en charge sur le côté $50 \mathrm{kV}$. permettent des variations de $\pm 8,7 \%$ en \pm 8 échelons.

Leur cuve est ondulée et le refroidissement est naturel jusqu'à $55 \%$ de la pleine charge. Au-dessus, les transformateurs sont souffiés par deux groupes de 6 ventilateurs.

Le raccordement au poste $50 \mathrm{kV}$. est réalisé en câbles sous huile de $50 . \mathrm{m}$. de longueur.

Le transformateur de couplage $50 / 10 \mathrm{kV}$. de $1.800 \mathrm{kVA}$. (puissance qui pourra ultérieurement ètre portée à $2.500 \mathrm{kVA}$. par souffiage) et les deux transformateurs des services auxiliaires de $125 \mathrm{kVA}$. sont installés à l'intérieur.

La dépense totale de l'installation s'est élevée à 1.065.000 R. M.

B. Koetzold.

Revue Liemens, no 2, 1934.

\section{Installation électrique de $200.000 \mathrm{~kW}$ pour l'électro-chimie en Corée}

La région de la Corée est très montagneuse. Parmi les nombreuses rivières, le Fousenko a été aménagé pour fournir l'énergie à la Société Coréenne des engrais azotés artificiels.

Grâce à un barrage de $80 \mathrm{~m}$. de hauteur on a pu créer une accumulation de 670 millions de $\mathrm{m}^{3}$ dont 478 millions de $\mathrm{m}^{3}$ utiles, située à $1200 \mathrm{~m}$. d'altitude, avec un débit disponible moyen de $23 \mathrm{~m}^{3} / \mathrm{sec}$. La pente de la vallée du Fousenko étant trop faible, on a reconnu plus économique de construire les usines hydro-électriques sur l'autre versant de la montagne où coule la rivière Yosenko. Il a fallu pour cela percer un tunnel de $26,6 \mathrm{~km}$. de longueur qui débourhe $10 \mathrm{~km}$. en amont du barrage placé sur l'autre vallée.

La première centrale est celle de Shoko $n^{\circ} 1$ construite près de Sokori. La chute brute est de $737 \mathrm{~m}$. I'usine comprend 4 groupes comprenant chacun une turbinc de $45000 \mathrm{CV}$. et un alternateur de $32400 \mathrm{~kW}$, à axe horizontal. Chaque groupe travaille séparément avec un transformateur correspondant de $36000 \mathrm{kVA} .11 / 110 \mathrm{kV}$.

L'usine de Shoko $\mathrm{n}^{\circ} 2$ utilise une chute de $215 \mathrm{~m}$..

Elle comprend deux groupes à axe vertical formés d'une tur- bine de $31000 \mathrm{CV}$. et d'un alternateur de $20700 \mathrm{~kW}$. et de 2 transformateur's de $23000 \mathrm{kV \Lambda} .11 / 110 \mathrm{kV}$.

Ensuite vient la centrale de Toko, près de Tokori qui, avec $93,2 \mathrm{~m}$. de chute possède une seule unité de $27000 \mathrm{GV}, 18000 \mathrm{~kW}$. et 3 transformateurs de $6666 \mathrm{kV \Lambda}$. $11 / 110 / 63,6 \mathrm{kV}$.

Enfin la centrale de Shinko, près de Shozniri, utilise sous 40,6 m. de chute, l'eau des centrales précédentes augmentée de celle clu fleuve Yosenko. Elle a 2 turbines à axe vertical de $9000 \mathrm{CV}$. accouplés à rles alternateurs de $6000 \mathrm{~kW}$., et 3 transformateurs de $4333 \mathrm{kVA} .11 / 110 / 63,6 \mathrm{kV}$.

Tout le matériel destiné à l'équipement de ces centrales a été construit au Japon. Pour les alternateurs de $32400 \mathrm{~kW}$. de l'usine de Shoko $n^{\circ} 1$, étant donné que, pour des raisons de transport, on ne pouvait pas rlépasser des pièces de plus de 20 tonnes, chacun des rotors a été construit en trois pièces. Tout est prévu pour assurer une marche semi-automatique, ou aulomatique de ces centrales.

\section{W. Ammens}

E. T. Z. 18 Janvier 1934. 


\section{Résonnance de l'oscillation dans une chambre d'équilibre}

On se borne en général à envisager dans le calcul des chambres d'équilibre, deux manœuvres opposées : l'ouverture d'un ou de plusieurs groupes à partir du niveau le plus bas de retenue et la fermeture complète de tous les groupes en cas de court-circuit, quand le niveau de retenue est le plus haut.

On pourrait, en ouvrant et en fermant successivement les vannes à un intervalle correspondant à une demi-période d'oscillation, augmenter artificiellement l'amplitude. $\mathrm{Si}$ cette manouvre ne correspond à aucune fonctionnement normal d'une usine, par contre, deux cas peuvent se produire, où l'amplitude de l'oscillation peut être amplifiće par résonnance-:

Premier cas : Court-circuit général, suivi d'une ouverture complète. - La fermeture des vannes, par suite de la suppression brusque de la charge, provoque une oscillation. En remettant la centrale en service, on crée une seconde oscillation par l'ouverture des vannes. La résultante dépend de l'intervalle du temps qui sépare l'ouverture de la fermeture. Il faut donc éviter que l'amplitude ne dépasse celle calculée pour la fermeture complète, ou l'ouverture complète, n'opérer la réouverture complète, après un court-circuit général qu'après une période d'attente définie par le temps $T+n T$ (compté à partir du court-circuit) et dans un délai qui ne devrait pas excèder $\pm \frac{T}{6}, T$ étant la période d'oscillation
Il y a lieu de remarquer que dans le calcul on ne tient pas compte du débit des turbines à vide, ni des pertes de charges. De plus les turbines ne sont pas toutes chargées simultanément à la remise en marche et c'est ce qui explique pourquoi le maximum théorique ne se réalise pratiquement jamais.

Second cas : Ouverture complète, suivie d'un court-circuit général. - C'est l'inverse du premier cas, mais avec cette diffé rence que le court-circuit survient inopinément. Il n'y a pas d'autres moyen de parer à cet effet que de munir la chambre d'équilibre d'un déversoir à sa partie supérieure. Cependant la mise en charge complète instantanée de toute l'usine ne se réalise pratiquement jamais.

Les ouvertures successives et fermetures des turbines ne doivent se faire qu'avec un délai voisin de la période entière ou de la demi-période d'oscillation. Quant aux manœuvres rythmées, il y a lieu de les éviter absolument, car rien ne pourrait s'opposer à leurs effets désastreux. De telles manœuvres ne se présentent pas en exploitation. Tout au plus pourraient-elles constituer des essais de réception.

Jules CaLAme.

Bulletin Technique de la Suisse Romande. 26 mai - 9 juin 1934.

\section{Le béton vibré ou pervibré, ses propriétés et conditions d'emploi}

Pour un dosage donné la résistance d'un béton est d'autant plus grande que I'eau de gâchage est plus faible et que sa densité est plus grande. On a donc intérêt à utiliser un béton consistant; mais alors il est difficile même par damage d'éviter la formation de nids de graviers, de zones poreuses de moindre résistance. C'est pour cela que l'on utilise un béton très plastique.

Sous l'action de marteaux à air comprimé, ou de vibrateurs fixés rigidement aux coffrages, le béton se liquéfie, remplit tous les vides et assure un enrobement parfait des armatures. Ce procédé a été employé avec succès pour le viaduc de Plougastel et pour le pont-route du Guenroz sur le Trient.

Du fait de la réduction de la quantité d'eau de gâchage la vibration a permis d'obtenir, avec la même facilité de mise en œuvre, des bétons ayant une résistance d'environ $50 \%$ plus élevée que ceux, de même dosage et fabriqués avec même ballast, à la consistance plastique habituelle. Mais la vibration ne permet de remédier que dans une faible mesure à un excès d'eau de gâchage initial.

La vibration se transmet le long des coffrages sur une distance qui ne dépasse pas $1 \mathrm{~m}$. et sur une profondeur de 20 à $30 \mathrm{~cm}$. pour le béton à consistance de terre humide, de 30 à $50 \mathrm{~cm}$. pour le béton plastique.

La vibration sur les coffrages ne convient pas pour la mise en œuvre de grosses masses de béton, on a essayé la pervibration qui consiste à mettre des vibrateurs flottants à la surface du béton. Mais ce procédé n'est pas encore au point.

\section{J. Bolomey.}

Bulletin Technique de la Suisse Romande. 14 avril 1934.

\section{Le développement des véhicules électriques à accumulation, à Lyon}

La ville de Lyon, qui avait décidé en 1924 de créer un service de transport en commun au moyen d'autobus à accumulateurs électriques, a dû, pour faire face aux besoins du public, augmenter progressivement son effectif et actucllement dispose de 62 véhicules, dont 10 à essence.

Les nouveaux autobus de la Société Vétra comportent des amèliorations intéressantes.

Le châssis est à deux essieux : celui avant est directeur, celui arrière moteur. Le poids du véhicule à vide, en ordre de marche, y compris la batterie d'accumulateurs, du poids de 3.000 kilos, pèse cnviron 8.300 kilos.

La construction du châssis est faite suivant la technique employée dans la construction automobile. Les freins sont au nombre de trois : $1^{\circ}$ un frein à main sur les roues arrières; $2^{\circ}$ un frein à pédale, qui agit sur les 4 roues, par l'intermédiaire d'un servofrein mécanique; $3^{\circ}$ un dispositif de récupération d'énergie électrique.

Le moteur électrique est du type campound à 4 pôles principaux et 4 pôles auxiliaires. La batteric d'accumulateurs comprend 42 éléments au plomb, d'une capacité de 800 ampères-heure.

La carrosserie comporte : un poste de conduite avant où prend place le conducteur et au besoin un agent de service; une petite plateforme avant fermée, où peuvent prendre place 10 voyageurs debout et 4 voyageurs assis ; à la suite, disposées de part et d'autre d'un couloir central, se trouvent dix banquettes de deux places chacune; une plateforme arrière ouverte, pour 12 voyageurs debout. L'accès à cette plateforme s'effectue par une entréc, placée à $45^{\circ}$, dans l'angle arrière droit, très commode pour l'accès de la voiture.

Les essais ont montré que la consommation moyenne en wattsheure, à la tonne kilomètrique diminue, quand la charge utile augmente. Elle augmente de $22 \%$ sur rampe de $3 \%$ et de $58 \%$ sur rampe de $5 \%$, la vitesse passant de $23,33 \mathrm{~km} / \mathrm{h}$ à 18,40 et 12,25 pour rampe de 3 et $5 \%$.

Le kilomètre lancé a fait ressortir une vitesse moyenne de 33,735 $\mathrm{km} / \mathrm{h}$. En service urbain la consommation moyenne à la tonne kilométrique est de 61,19 Wh., avec une vitesse commerciale de $16,25 \mathrm{~km} / \mathrm{h}$, arrèts compris. Un essai sur le parcours Lyon-Bourgoin, aller et retour, soit $78,8 \mathrm{~km}$., fait à une vitesse normale d'exploitation et économique, a accusé une consommation remarquable de $39 \mathrm{Wh}$. à la tonne kilomètrique. La batterie de $800 \mathrm{Ah}$ au départ pouvait encore débiter à l'arrivée à Lyon, 380 Ah. Le coefficient de roulement a été trouvé égal à $12,88 \mathrm{~kg}$ partonne.

Le kilométrage moyen, assuré chaque jour de semaine, est actuellement de $4.850 \mathrm{~km}$., pour l'ensemble du réseau en service. Des prolongements de certaines lignes sont projetés.

\section{Chaldumeau.}

La Traction Electrique, mars 1934. 